\title{
Facklamia hominis bacteremia after transurethral resection of the prostate: a case report
}

\author{
Miriam Gahl ${ }^{1}$, Thomas Stöckli ${ }^{2}$ and René Fahrner ${ }^{1 *}$ (D)
}

\begin{abstract}
Background: Transurethral resection of the prostate (TUR-P) is one of the most frequent routine procedures in urology. Because of the semisterile environment, postoperative infections, including sepsis, are a common complication, with Escherichia coli, Klebsiella spp., Proteus mirabilis or Enterococcus faecalis as frequently isolated pathogens. Facklamia hominis is a gram-positive, facultatively anaerobic, alpha-hemolytic, catalase-negative coccus that was first described in 1997. To date, only a few cases of infectious complications have been described. We report the first case of postoperative bacteremia due to Facklamia hominis after TUR-P.

Case presentation: An 82-year-old man developed fever only a few hours after elective TUR-P because of benign prostate syndrome. After cultivation of blood cultures, antibiotic therapy with ceftriaxone was intravenously administered and changed to oral cotrimoxazole before discharge of the afebrile patient. One anaerobic blood culture revealed Facklamia hominis. Under antibiotic therapy, the patient remained afebrile and showed no signs of infections during follow-up.

Conclusions: Fever and bacteremia are frequent complications after TUR-P. This study is the first report of Facklamia hominis in a postoperative blood culture after TUR-P. To date, there are only a few reports of patients with infectious complications and isolation of Facklamia hominis in various patient samples. Because Facklamia hominis resembles viridans streptococci on blood agar analysis, this pathogen may often be misidentified. In this case identification of Facklamia hominis was possible with matrix-assisted laser desorption/ionization time-of-flight mass spectrometry. It has been postulated that Facklamia hominis might be a facultative pathogen and that its incidence will increase in the future.
\end{abstract}

Keywords: Bacteremia, Facklamia hominis, Transurethral resection of the prostate, Genital flora

\section{Background}

Transurethral resection of the prostate (TUR-P) is one of the most frequent routine procedures in urology. Because of the semisterile environment and continuous rinsing with water, postoperative infections, including sepsis, are a common complication [1,2]. Despite this

\footnotetext{
${ }^{*}$ Correspondence: r.fahrner@web.de

1 Department of General, Visceral and Thoracic Surgery, Bürgerspital

Solothurn, Schöngrünstrasse 42, 4500 Solothurn, Switzerland

Full list of author information is available at the end of the article
}

fact, prophylactic antibiotics are still controversial, and applied substances vary in regard to the local spectrum of bacteria [1-4]. As a sign of procedural bacteremia, postoperative fever is often encountered early after surgical intervention. The most frequently isolated pathogens are Escherichia coli, Klebsiella spp., Proteus mirabilis and Enterococcus faecalis, which are also often detected during simple cystitis [5]. To cover these pathogens, preoperative prophylaxis with a single dose of oral ciprofloxacin is usually given in our department. In a case of postoperative urinary tract infection, daily ceftriaxone is 
administered intravenously, with a change to oral treatment before discharge. The antibiotic prophylaxis regarding the guidelines of the European Association of Urology should include the local pathogen prevalence and might thus differ from center to center [6].

To the best of our knowledge, we present the first case of Facklamia hominis bacteremia during the postoperative course after urological surgery.

\section{Case presentation}

An 82-year-old man underwent elective TUR-P because of a symptomatic benign prostate syndrome. Preoperatively, no urinary sample was analyzed regarding bacterial colonization. He had a past history of cerebrovascular insult with minimal residuals, curative surgery for an adenocarcinoma of the rectum and cervical discus hernia. In addition, he suffered from hypertensive cardiopathy with a normal ejection fraction.

One hour after an uneventful operation, he developed chills that were successfully treated with pethidine. Three hours later, he developed a fever up to $38.7^{\circ} \mathrm{C}$ so that two pairs of blood cultures were taken before initiating intravenous antibiotic therapy with ceftriaxone. Because of postoperative continuous rinsing of the bladder, it was impossible to cultivate the urine. The further course was uneventful, the patient remained afebrile and was in good condition so that the antibiotic therapy was changed to oral cotrimoxazole, and the patient was discharged. To our surprise, one out of four blood cultures turned positive for Facklamia hominis after the discharge of the patient. As the patient remained afebrile and in good clinical condition under the current antibiotic treatment, the therapy was continued for 14 days, although cotrimoxazole has not been described as a therapy so far. During 6 months of follow-up, the patient did not develop fever or signs of an urinary tract infection and had no need for antibiotic therapy again. During follow-up, there were urine and blood cultures without detection of Facklamia hominis.

\section{Discussion and conclusions}

In our case, bacteremia with Facklamia hominis was detected in blood cultures of a patient after TUR-P only a few hours after the intervention. Facklamia hominis is a gram-positive, facultative anaerobic, alpha-hemolytic, catalase-negative coccus [7]. It was first described in 1997 [8], and for six clinical isolates, previously nonclassified cocci were characterized by phenotypic and phylogenetic methods as Facklamia hominis [9]. Since then, Facklamia hominis has been isolated in several specimens, such as urine, vaginal swabs, abscesses, joints, mitral valves, placentas, gastric aspirates, cerebrospinal fluid, preputial swabs, and blood [10-15].Moreover, Facklamia hominis is thought to be a resident of the bacterial flora of the vaginal and urinary tracts [14]. Interestingly, Facklamia hominis has been so far isolated predominantly from females. In total, 16 cases of Facklamia hominis infections have been reported worldwide (Table 1). Furthermore, five other species of the genus Facklamia have been described, namely, Facklamia ignava [17], Facklamia sourekii [18], Facklamia languida [19], Facklamia miroungii [20] and Facklamia tabaciasalis [21]. Except for Facklamia tabaciasalis [21] and Facklamia miroungii [20], all Facklamia species have been isolated from human clinical specimens. It is postulated that, as Facklamia spp. resemble viridans streptococci on 5\% sheep blood agar, they might have been confounded in the past with this group of organisms $[9,16]$.

This study is the first case reported with Facklamia hominis bacteremia after TUR-P. There have been reports about isolations from patients with abscesses, joint infections, endocarditis with positive blood cultures, cerebrospinal fluid, urine and vaginal swabs (Table 1). It has been postulated that Facklamia

Table 1 Overview of Facklamia hominis infections in the current literature

\begin{tabular}{llll}
\hline Author & Year & Number of patients & Type of sample \\
\hline Collins [8] & 1997 & 6 & Urine, vaginal swab, blood, abscess \\
Healy [10] & 2005 & 2 & Blood, placental tissue, gastric aspirate \\
Safavi [11] & 2010 & 1 & Blood \\
Ananthakrishna [16] & 2012 & 1 & Blood \\
Corona [7] & 2014 & 1 & Joint \\
Parvataneni [9] & 2015 & 1 & Cerebrospinal fluid \\
Abat [15] & 2016 & 1 & Abscess \\
Schlipkoter [12] & 2017 & 1 & Abscess \\
Gomez-Luque [13] & 2019 & 1 & Cured \\
Mostafa [14] & 2019 & 1 & Cured \\
\hline
\end{tabular}


hominis might be a resident of the vaginal and urinary tract floras and a facultative pathogen inducing urinary tract infections [14]. In the reported case, the source of Facklamia hominis is speculative and might be displaced during surgery from the urinary tract or urine. Furthermore, the prostate might be colonized, but microscopy of the surgical tissue failed to detect large amount of bacteria. Fever or infections after TUR-P are frequently seen complications, as the intervention is semisterile, and microorganisms located within the urinary tract are often opportunistic. These facultative pathogens are common sources of postoperative bacteremia or urinary tract infections $[1,2]$. Accordingly, it is not surprising that Facklamia hominis was now isolated in blood cultures after TUR-P. The treatment includes immediate antibiotic therapy depending on the prevalent resistance pattern after cultivation of blood and urine. To obtain an optimal antibiotic therapy, the isolation of the underlying pathogens is mandatory.

Traditional microbiological methods are often ineffective in correctly detecting pathogens such as Facklamia hominis [15]. Current methods of identification include matrix-assisted laser desorption/ionization time-of-flight mass spectrometry (MALDI-TOF) [13, 15], genome sequencing [14], rRNA PCR [7], VITEK 2 system $[9,16]$ and characterization by the API Rapid ID32 and API ZYM method [8]. Comparable to previous reports, in our case, Facklamia hominis was detected by MALDI-TOF.

Several treatment regimens have been described so far. They include penicillin derivatives, beta-lactamase inhibitors, metronidazole, cephalosporins, carbapenems, aminoglycosides, and glycopeptide antibiotics [7, 10-12, 16]. In our case, the intravenous treatment with ceftriaxone for 3 days and cotrimoxazole for a total of 14 days was successful. Whether the intravenous administration of ceftriaxone alone would have been sufficient as treatment is unclear. However, the patient rapidly recovered and remained afebrile without signs of bloodstream or urinary tract infection.

It is possible that due to the morphologic resemblance to viridans streptococci and ineffectiveness of traditional microbiological testing, Facklamia hominis has probably been often misdiagnosed in the past [15]. Whether Facklamia hominis will be an emerging pathogen in the future needs to be confirmed, but additional reports on antibiotic therapy are needed.

\footnotetext{
Abbreviations

C: Celcius; MALDI-TOF: Matrix-assisted laser desorption/ionization time-offlight mass spectrometry; spp.: species; TUR-P: Transurethral resection of the prostate.
}

Acknowledgements

Not applicable for this study.

\section{Authors' contributions}

Study conception and design: M. G., T. S., and R. F. Acquisition of data: M. G., T. S., and R. F. Analysis and interpretation of data: M. G., T. S., and R. F. Drafting of manuscript: M. G., and R. F. Critical revision of manuscript: T. S. All authors have read and approved the final version of this manuscript.

Funding

Not applicable for this study.

\section{Availability of data and materials}

The datasets used and/or analyzed during the current study are available from the corresponding author on reasonable request.

\section{Ethics approval and consent to participate}

All research protocols were carried out in accordance with the National Institutes of Health guidelines.

\section{Consent for publication}

Written consent to publish this case presentation was obtained from the patient.

\section{Competing interests}

René Fahrner is a member of the editorial board (Section Editor) of BMC Surgery. The other authors declare that they have no conflicts of interest.

\section{Author details}

${ }^{1}$ Department of General, Visceral and Thoracic Surgery, Bürgerspital Solothurn, Schöngrünstrasse 42, 4500 Solothurn, Switzerland. ${ }^{2}$ Department of Internal Medicine, Bürgerspital Solothurn, Schöngrünstrasse 42, 4500 Solothurn, Switzerland.

Received: 17 September 2020 Accepted: 25 November 2020

Published online: 07 December 2020

\section{References}

1. Schneidewind L, Kranz J, Schlager D, Barski D, Muhlsteadt S, Grabbert M, Queissert F, FrankT, Pelzer AE. Mulitcenter study on antibiotic prophylaxis, infectious complications and risk assessment in TUR-P. Central Eur J Urol. 2017;70(1):112-7.

2. Wagenlehner FM, Wagenlehner C, Schinzel S, Naber KG, Working group "urological infections" of German Society of U. Prospective, randomized, multicentric, open, comparative study on the efficacy of a prophylactic single dose of $500 \mathrm{mg}$ levofloxacin versus $1920 \mathrm{mg}$ trimethoprim/sulfamethoxazole versus a control group in patients undergoing TUR of the prostate. Eur Urol. 2005;47(4):549-56.

3. Schmiedl S, Thurmann PA, Roth S. Antibiotic prophylaxis for patients with transurethral resection of the prostate (TUR-P). Der Urol Ausg A. 2009;48(1):66-72.

4. Berry A, Barratt A. Prophylactic antibiotic use in transurethral prostatic resection: a meta-analysis. J Urol. 2002;167(2 Pt 1):571-7.

5. Hautmann R, Gschwend JE. Urologie. Springer Medizin Verlag. 2014. https $\cdot / /$ doi.org/10.1007/978-3-642-34319-3.

6. https://uroweb.org/guideline/urological-infections/. Accessed 07 Nov 2020.

7. Corona PS, Haddad S, Andres J, Gonzalez-Lopez JJ, Amat C, Flores X. Case report: first report of a prosthetic joint infection caused by Facklamia hominis. Diagn Microbiol Infect Dis. 2014;80(4):338-40.

8. Collins MD, Falsen E, Lemozy J, Akervall E, Sjoden B, Lawson PA. Phenotypic and phylogenetic characterization of some Globicatella-like organisms from human sources: description of Facklamia hominis gen. Nov., sp. nov. Int J Syst Bacteriol. 1997:47(3):880-2.

9. Parvataneni KC, Iyer S, Khatib R, Saravolatz LD. Facklamia Species and Streptococcus pneumoniae Meningitis: A Case Report and Review of the Literature. Open forum Infect Dis. 2015;2(2):ofv029.

10. Healy B, Beukenholt RW, Tuthill D, Ribeiro CD. Facklamia hominis causing chorioamnionitis and puerperal bacteraemia. J Infect. 2005;50(4):353-5.

11. Safavi S, Tufnell M, Bhalla A. Multi-territory ischaemic strokes and subacute bacterial endocarditis. BMJ Case Rep. 2010;2010. 
12. Schlipkoter M, Grieser T, Forst H. Unusual complication following placement of an epidural catheter. Anaesthesist. 2017;66(7):506-10.

13. Gomez-Luque JM, Foronda-Garcia-Hidalgo C, Gutierrez-Fernandez J. Balanopostitis by Facklamia hominis in pediatrics. Revista espanola de quimioterapia. 2019;32(3):278-80

14. Mostafa HH, Taffner SM, Wang J, Malek A, Hardy DJ, Pecora ND. Genome sequence of a Facklamia hominis isolate from a patient with Urosepsis. Microbiol Resource Announcements. 2019;8(17).

15. Abat C, Garcia V, Rolain JM. Facklamia hominis scapula abscess, Marseille, France. New Microbes New Infect. 2016;9:13-4.

16. Ananthakrishna R, Shankarappa RK, Jagadeesan N, Math RS, Karur S, Nanjappa MC. Infective endocarditis: a rare organism in an uncommon setting. Case Rep Infect Dis. 2012;2012:307852.

17. Collins MD, Lawson PA, Monasterio R, Falsen E, Sjoden B, Facklam RR. Facklamia ignava sp. nov., isolated from human clinical specimens. J Clin Microbiol. 1998;36(7):2146-8.
18. Collins MD, Hutson RA, Falsen E, Sjoden B. Facklamia sourekii sp. nov., isolated from human sources. Int J Syst Bacteriol. 1999;49(Pt 2):635-8.

19. Lawson PA, Collins MD, Falsen E, Sjoden B, Facklam RR. Facklamia languida sp. nov., isolated from human clinical specimens. J Clin Microbiol. 1999;37(4):1161-4.

20. Hoyles L, Foster G, Falsen E, Thomson LF, Collins MD. Facklamia miroungae sp. nov., from a juvenile southern elephant seal (Mirounga leonina). Int J Syst Evol Microbiol. 2001;51 (Pt 4):1401-3.

21. Collins MD, Hutson RA, Falsen E, Sjoden B. Facklamia tabacinasalis sp. nov., from powdered tobacco. Int J Syst Bacteriol. 1999;49(Pt 3):1247-50.

\section{Publisher's Note}

Springer Nature remains neutral with regard to jurisdictional claims in published maps and institutional affiliations.
Ready to submit your research? Choose BMC and benefit from:

- fast, convenient online submission

- thorough peer review by experienced researchers in your field

- rapid publication on acceptance

- support for research data, including large and complex data types

- gold Open Access which fosters wider collaboration and increased citations

- maximum visibility for your research: over $100 \mathrm{M}$ website views per year

At BMC, research is always in progress.

Learn more biomedcentral.com/submissions 